\title{
Frequency of SMN1 deletion carriers in a Mestizo population of central and northeastern Mexico: A pilot study
}

\author{
SILVINA NOEMI CONTRERAS-CAPETILLO ${ }^{1}$, HUGO LEONID GALLARDO BLANCO ${ }^{2}$, \\ RICARDO MARTIN CERDA-FLORES ${ }^{3}$, JOSÉ LUGO-TRAMPE ${ }^{2}$, IRIS TORRES-MUÑOZ ${ }^{2}$, \\ ANTONIO BRAVO-ORO ${ }^{4}$, CARMEN ESMER $^{5}$ and LAURA ELLA MARTÍNEZ DE VILLARREAL ${ }^{2}$ \\ ${ }^{1}$ Department of Genetics, Dr. Hideyo Noguchi Regional Research Center, Autonomous University of Yucatan, \\ Mérida, Yucatán CP 97225; ${ }^{2}$ Department of Genetics, School of Medicine; ${ }^{3}$ School of Nursing, \\ Autonomous University of Nuevo León, Monterrey, Nuevo León CP 64460; \\ Departments of ${ }^{4}$ Neuropediatrics and ${ }^{5}$ Genetics, Central Hospital 'Dr. Ignacio Morones Prieto', \\ San Luis Potosí CP 78240, Mexico
}

Received July 4, 2014; Accepted March 16, 2015

DOI: $10.3892 / \mathrm{etm} .2015 .2436$

\begin{abstract}
Individuals who suffer from spinal muscular atrophy (SMA) exhibit progressive muscle weakness that frequently results in mortality in the most severe forms of the disease. In $98 \%$ of cases, there is a homozygous deletion of the survival of motor neuron 1 (SMN1) gene, and both parents carry the same heterozygous genetic abnormality in the majority of cases. Various population studies have been conducted to estimate the frequency of carriers and thereby identify the communities or countries in which children are at a high risk of being affected by SMA. However, the prevalence of SMA in Mexican populations has not yet been established. In the present pilot study, the frequency of the heterozygous deletion of the SMN1 gene was determined in two groups from northeastern $(n=287)$ and central $(n=133)$ Mexican Mestizo populations and compared with other ethnic populations. Amplification refractory mutation system polymerase chain reaction analysis yielded a disease carrier frequency of 11/420 (2.62\%) healthy individuals, comprising 9/287 (3.14\%) northeastern and 2/133 (1.5\%) central Mexican individuals. In summary, no significant differences were identified between the northeastern and central populations of Mexico and other ethnic populations, with the exception of the general worldwide Hispanic population, which exhibited the lowest carrier frequency of 8/1,030.
\end{abstract}

Correspondence to: Dr Hugo Leonid Gallardo Blanco, Department of Genetics, School of Medicine, Autonomous University of Nuevo León, Francisco I. Madero Avenue and José Eleuterio González Avenue, Colonia Mitras Centro, Monterrey, Nuevo León CP 64460, Mexico

E-mail: hugoleonid2011@me.com

Key words: spinal muscular atrophy type I, heterozygous carriers, hypotonic syndrome, SMN1 gene, neuromuscular diseases
The results of the present study may be used to improve the evaluation procedure, and appear to justify further studies involving larger sample populations.

\section{Introduction}

Spinal muscular atrophy (SMA) is an autosomal recessive disease that is a result of the degeneration and progressive death of $\alpha$ motor neurons in the anterior horns of the spinal cord and brainstem nuclei (1). SMA is characterized by progressive muscle weakness, and is the most common genetic cause of infant mortality (1). SMA presents with an extremely variable phenotype. The clinical spectrum of SMA is continuous; however, variations in the age of onset and symptoms have led to a classification system of the clinical variants of SMA that is based on maximum patient motor skill achievement. This classification system has resulted in an improved guideline design for the clinical management and follow-up of SMA, which is based on the patient's maximum motor skills $(2,3)$. Sixty percent of SMA cases are type I, which is characterized by proximal muscle weakness, affecting the legs most severely, areflexia, and abdominal breathing that produces a bell-shaped chest deformity with breathing complications. SMA type I is also known as Werdnig-Hoffman disease (OMIM, \#253,300) and has a prevalence of 4.1/100,000 live births, with patient mortality typically occurring at $\sim 2$ years of age (4). SMA type II, the intermediate form (OMIM, \#253,550) of the disease, has an onset age of 6-18 months. Patients with SMA type II may be able to sit, but develop hypotonia, hyporeflexia and lingual fasciculation, and $98 \%$ of affected individuals survive to 5 years of age, although overall lifespan is reduced $(2,3)$. SMA type III (Kugelberg-Welander type; OMIM, \#253,400) has an onset age of 18 months, and patients affected with this SMA type are able to sit and walk (5). SMA type IV presents in adults $>21$ years; these patients exhibit a normal acquisition of all fine motor skills prior to the onset of neuromuscular symptoms and usually have a normal lifespan (6). 
The overall prevalence of SMA is $1 / 6,000-1 / 10,000$, with a carrier frequency range of 1/40-1/60 $(7,8)$. Homozygous loss of the survival of motor neuron 1 (SMN1) gene accounts for 94-95\% of all SMA cases. The remaining $5 \%$ of cases consist of compound heterozygotes with the SMN1 deletion in one allele and an alternative mutation in the remaining allele. This mutational event occurs in $<1 / 4,000$ affected individuals and follows the Hardy-Weinberg equilibrium (9-11). The chromosomal region $5 \mathrm{q} 11.2-13.3$ is susceptible to non-allelic homologous chromosomal rearrangements. The two variants of the $S M N$ gene, a telomeric (SMN1 or $S M N T)$ (OMIM, $\# 600,354)$ and a centromeric gene $(S M N 2$ or $S M N c)(\mathrm{OMIM}$, \#601,627), differ in a single nucleotide $(840 \mathrm{C}>\mathrm{T})$, which results in the alternative splicing of exon 7 in the SMN2 gene in $75-90 \%$ of the transcripts produced $(2,12-16)$. The severity of SMA is primarily determined by the copy number of the SMN2 gene. The SMN protein forms a complex of molecules that regulates motoneuron survival by maintaining normal axonal transport and the growth, maturation and formation of axons and neurites (17-19).

SMA is diagnosed using various molecular procedures; the majority are polymerase chain reaction (PCR)-based methods and rely on an analysis of the gene dosage. In the present study, the frequency of SMN1 gene deletion was determined in healthy carriers and non-carriers from northeastern and central Mexican Mestizo populations. The results may aid in establishing a basis for implementing carrier screening programs across the country following further validation.

\section{Materials and methods}

This pilot study (no. GN08-005) was conducted at the Department of Genetics, School of Medicine, Autonomous University of Nuevo León (Monterrey, Mexico). The study was approved by the committee for ethics, research and biosecurity of the Autonomous University of Nuevo León.

A total of 420 individuals aged $>18$ years, with parents and four grandparents of Mexican origin, were recruited from northeastern $(n=287)$ and central Mexico $(n=133)$. Informed consent was obtained from all participants prior to peripheral blood collection by venipuncture in accordance with the ethical guidelines established by the Declaration of Helsinki of the World Medical Association in 1964 and modified in 1989. Genomic DNA was obtained from peripheral blood cells using a QIAamp ${ }^{\circledR}$ Blood Mini kit employing the automated system QIAcube (Qiagen GmbH, Hilden, Germany).

Molecular detection of the SMN1 deletion. A molecular assay was designed to simultaneously determine the relative gene dosage of the SMN1 deletion and SMN2 in relation to the TP53 gene, which was used as an internal control. This method was based on amplification refractory mutation system PCR (ARMS-PCR) (20) using six primers and capillary electrophoresis (Fig. 1). The four primers (Applied Biosystems Life Technologies, Foster City, CA, USA) used to amplify the SMN1 and SMN2 genes were as follows: SMN1, forward 5'-TGTGAAACAAAATGCTTTTTAACATCC-3' and reverse 5'-AAAACATTTGTTTTCCAAAACCAT AAA-3'; $S M N 2$, forward 5'-FAM-TTCCTTTATTTTCCT TACAGGGTGTC-3' and reverse 5'-FAM-CACCTTCCT
TCTTTTTGATTTTGTATA-3'. The primers used to amplify the TP53 gene were as follows: Forward 5'-GGTCCAGAT GAAGCTCCCAGAAT-3' and reverse 5'-FAM-TCACAG ACTTGGCTGTCCCAGAAT-3'. Primers labeled 5'-FAM were 5 ' fluorescently labeled with the fluorophore 6-FAM.

The method was validated by assaying triplicate DNA samples from six individuals with SMA type I that were homozygous for the deletion of the SMN1 gene, based on a PCR-restriction fragment length polymorphism analysis. In addition, samples from healthy carriers of the deletion were used in the validation assay.

PCR procedure. PCR reactions were conducted in a reaction system containing $20 \mathrm{ng}$ genomic DNA, $0.031 \mu \mathrm{M}$ TP53 primers, $0.038 \mu \mathrm{M}$ forward and $0.42 \mu \mathrm{M}$ reverse external primers for $S M N 1 / S M N 2,0.022 \mu \mathrm{M}$ primer specific to the 'C' nucleotide (c.840C of SMN1), $0.030 \mu \mathrm{M}$ primer specific to the ' $\mathrm{T}$ ' nucleotide (c.840T of SMN2), $0.75 \mathrm{U}$ GoTaq DNA polymerase (Promega Corporation, Madison, WI, USA ), $1.5 \mathrm{mM} \mathrm{MgCl}_{2}$ and $0.3 \mathrm{mM}$ dNTPs (Promega Corporation), with a final volume of $15 \mu$ l. The cycling procedure included one cycle of initial denaturation at $95^{\circ} \mathrm{C}$ for $1 \mathrm{~min}, 29$ cycles of denaturation at $95^{\circ} \mathrm{C}$ for $40 \mathrm{sec}$, annealing at $53^{\circ} \mathrm{C}$ for $40 \mathrm{sec}$ and extension at $72^{\circ} \mathrm{C}$ for $1 \mathrm{~min}$; and a final $72^{\circ} \mathrm{C}$ extension step for $20 \mathrm{~min}$. Immediately, $1 \mu \mathrm{l}$ of the amplicon was mixed with $0.3 \mu$ l GeneScan ${ }^{\mathrm{TM}}-500$ LIZ $^{\circledR}$ Size Standard (Applied Biosystems Life Technologies) and $8.7 \mu 1$ formamide. This mixture was placed in a Hybaid PCR Express HBPX110 thermocycler (Thermo Fisher Scientific, Renfrew, UK) for $5 \mathrm{~min}$ at $95^{\circ} \mathrm{C}$, then at $-20^{\circ} \mathrm{C}$ for $5 \mathrm{~min}$. Following denaturation, the samples were analyzed using a 3100 Avant Genetic Analyzer (Applied Biosystems Life Technologies).

Estimation of gene copy number. Three peaks corresponding to the SMN2 (135 bp), SMN1 (149 bp) and TP53 (194 bp) genes were obtained using electropherograms generated by ABI Prism 3100 AVANT genetic analyzer (Applied Biosystems Life Technologies) (Fig. 2). The area of each peak was determined based on the GeneMapper software, version 3.1 with a peak detection algorithm (Applied Biosystems Life Technologies) and following a previously described protocol (21) with modifications (allele size detection adjusted for SMN1, SMN2 and TP53 amplicons). The ratio of the peak area of the SMN1 gene to the TP53 gene represented the relative copy number of the target gene SMN1. For this analysis, a TP53 gene dosage of $100 \%$ (two gene copies of TP53) was assumed to be present in all subjects, as none presented with Li-Fraumeni syndrome (22). Based on this analysis, subjects with at least two copies of SMN1 were considered to be healthy non-carriers, those with one copy of SMN1 were considered true carriers, and those with no copies of the SMN1 gene were considered to be affected with SMA. A limitation of this detection method is that it did not distinguish whether the two copies of the SMN1 gene were in cis or trans.

Statistical analysis. Fisher's exact test was applied to compare the northeastern and central Mexican Mestizo populations. The $\chi^{2}$ test was applied to compare the carrier frequency among the populations in the present study, worldwide populations and the Hispanic population. 
A

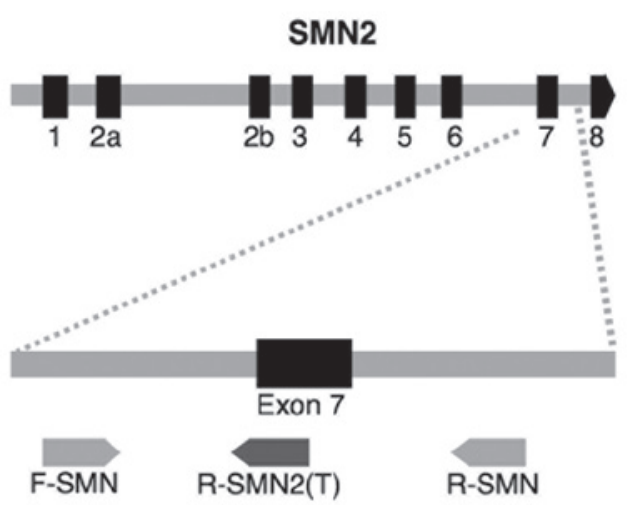

B

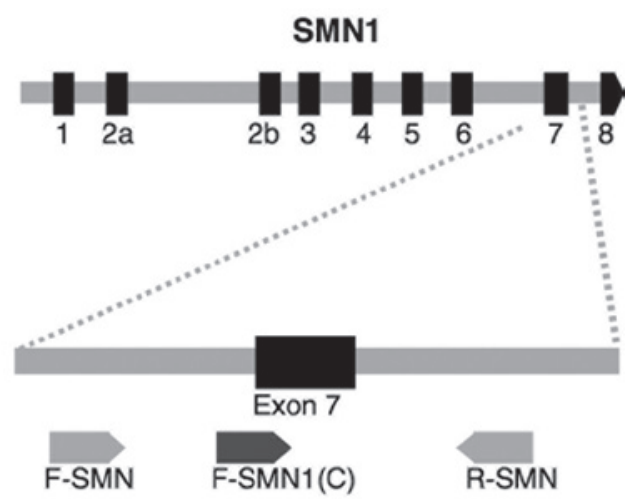

C

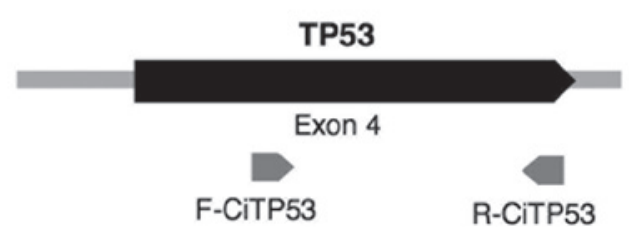

Figure 1. Primer binding in the SMN1, SMN2 and TP53 genes. The primer (A) R-SMN2 binds specifically to exon 7 in $S M N 2$ and (B) F-SMN1 binds specifically to exon 7 in SMN1. (C) Binding region of primers in the TP53 gene for copy number reference control. F, forward; R, reverse; SMN, survival of motor neuron.

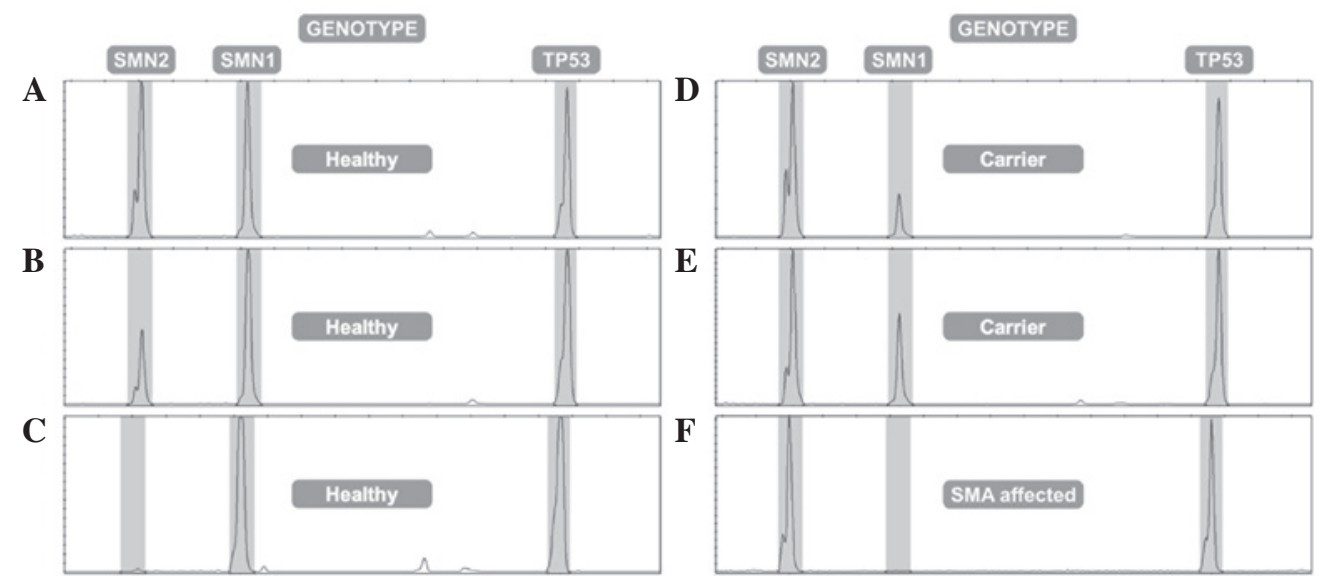

Figure 2. Electropherogram profiles of the affected, heterozygote and healthy individuals. (A, B and C) The presence of a SMN1 gene peak equal to TP53 indicates a healthy individual even in the presence of the SMN2 gene deletion. (D and E) The peak for SMN1 is half the size of the peak for the TP53 gene, which indicates the presence of a single copy of the SMN1 gene. (F) The absence of a peak indicates a homozygous deletion of SMN1 affected with spinal muscular atrophy. SMN1, survival of motor neuron 1.

Data were analyzed using SPSS statistical software, version 22.0 (IBM SPSS, Armonk, NY, USA). P $<0.05$ was considered to indicate a statistically significant difference.

\section{Results}

A total of 420 DNA samples were obtained from male and female participants aged 18-71 years, with four grandparents of Mexican descent. Among this population, $68.33 \%(\mathrm{n}=287)$ were from northeastern and $31.67 \%(n=133)$ from central Mexico. The SMN1 copy number was standardized using DNA from affected and healthy carrier individuals. The peak electropherogram area of the SMN1 gene in the affected patients was $0.00-0.05$, indicating a mean loss of the SMN1 gene among homozygous carriers. In heterozygous carriers, the range of the peak area was $0.3-0.52$, representing half of the subjects. The range was $>0.6$ for healthy individuals, which indicated complete gene dosage (Fig. 3). If it was not possible to determine whether a sample corresponded to a carrier or a non-carrier (SMN1/TP53 ratio between 0.52-0.6), the sample was reanalyzed in triplicate, and the mean of the three measurements was used.

Among the study population, 11 subjects $(2.62 \%)$ carried one copy of SMN1 and 409 subjects $(97.38 \%)$ carried two copies (Table I). Nine of the single-copy carriers (3.14\%) were from northeastern and two $(1.50 \%)$ were from central Mexico. No significant difference was observed between these groups using two-tailed Fisher's exact test $(\mathrm{P}=0.5143$; Table I). The overall frequency of carriers among the two Mexican population groups was $11 / 420$ (or $1 / 38$ ). Thus, the carrier frequency detected in the present pilot study was $2.62 \%$. 
Table I. Frequencies of SMN1 gene deletion carriers.

\begin{tabular}{|c|c|c|c|c|c|c|}
\hline \multirow[b]{2}{*}{ Carrier status } & \multicolumn{2}{|c|}{ Northeastern } & \multicolumn{2}{|c|}{ Central } & \multicolumn{2}{|c|}{ Total } \\
\hline & $\mathrm{n}$ & $\%$ & $\mathrm{n}$ & $\%$ & $\mathrm{n}$ & $\%$ \\
\hline Carriers & 9 & 3.14 & 2 & 1.50 & 11 & 2.62 \\
\hline Non-carriers & 278 & 96.86 & 131 & 98.50 & 409 & 97.38 \\
\hline Total & 287 & & 133 & & 420 & \\
\hline
\end{tabular}

Fisher's exact test, two-tailed $\mathrm{P}=0.5143$. SMN1, survival of motor neuron 1.

Table II. Distribution of carrier frequencies with an SMN1 deletion among nine populations worldwide and in the present study.

\begin{tabular}{|c|c|c|c|c|c|}
\hline \multirow[b]{2}{*}{ Population } & \multicolumn{2}{|c|}{ Carriers } & \multirow[b]{2}{*}{ Total } & \multirow[b]{2}{*}{ Method } & \multirow[b]{2}{*}{ Reference } \\
\hline & Yes $(\%)$ & No $(\%)$ & & & \\
\hline Mexican & $11(2.62)$ & $409(97.38)$ & 420 & PCR-ARMS & Present study \\
\hline Hispanic & $8(0.8)$ & $1,022(99.2)$ & 1,030 & RT-qPCR & Hendrickson et al, 2009 (4) \\
\hline African-American & $18(1.8)$ & $997(98.2)$ & 1,015 & RT-qPCR & Hendrickson et al, 2009 (4) \\
\hline Asian & $18(1.8)$ & $1,009(98.2)$ & 1,027 & RT-qPCR & Hendrickson et al, 2009 (4) \\
\hline Korean & $2(2.0)$ & $98(98.0)$ & 100 & MLPA & Yoon et al, 2010 (19) \\
\hline Jewish & $22(2.2)$ & $980(97.8)$ & 1,002 & RT-qPCR & Hendrickson et al, 2009 (4) \\
\hline Israeli & $159(2.5)$ & $6,235(97.5)$ & 6,394 & MLPA & Ben-Shachar et al, 2011 (23) \\
\hline Caucasian & $28(2.7)$ & $1,000(97.3)$ & 1,028 & RT-qPCR & Hendrickson et al, 2009 (4) \\
\hline Arabian & $2(4.0)$ & $48(96.0)$ & 50 & Multiplex-PCR & Majumdar et al, 2005 (8) \\
\hline Iranian & $10(5.0)$ & $190(95.0)$ & 200 & qPCR & Hasanzad et al, 2009 (9) \\
\hline Total & 278 & $12,08012,358$ & & & \\
\hline
\end{tabular}

$\chi^{2}=22.74, \mathrm{df}=9, \mathrm{P}=0.007$ (among 10 populations). $\chi^{2}=10.84, \mathrm{df}=8, \mathrm{P}=0.211$ (Hispanic population was not included). PCR-ARMS, polymerase chain reaction-amplification refractory mutation system; RT-qPCR, reverse transcription-quantitative polymerase chain reaction; MLPA, multiplex ligation-dependent probe amplification; SMN1, survival of motor neuron 1.
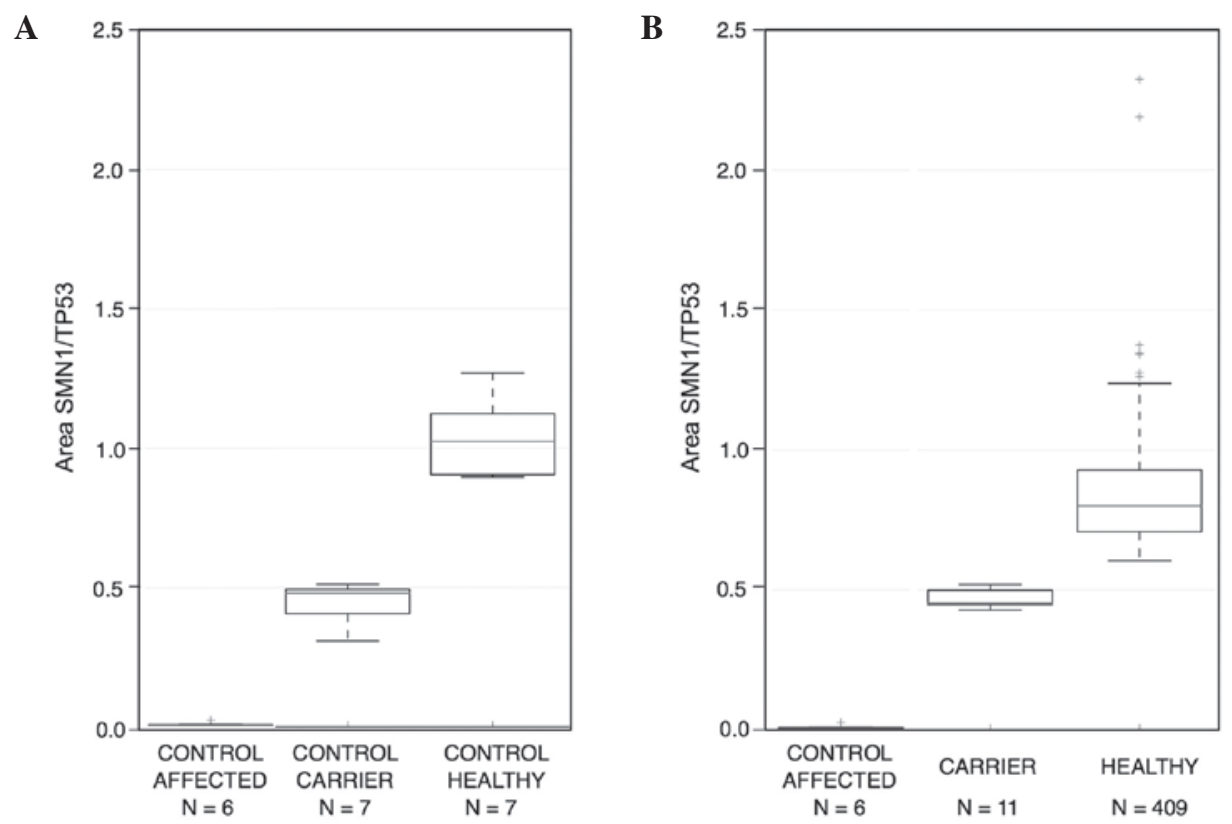

Figure 3. (A) Copy number validation of the SMN1 gene using the polymerase chain reaction-amplification refractory mutation system method. (B) Copy number determination for the 420 screened individuals presenting the ratio of peak area values of SMN1/TP53. Individuals are distributed around ratios of 0.0-0.05, 0.3-0.52 and >0.6, which represent 0, 1, and $\geq 2$ copies, respectively, of SMN1 exon 7. SMN1, survival of motor neuron 1. 


\section{Discussion}

The results of the present pilot study indicate that the carrier frequency of $S M N 1$ in northeastern and central Mexico is $1 / 38$, which is comparable to the reported frequencies in other ethnic populations, including African-American, Asian, Korean, Jewish and Israeli populations (Table II). However, the SMA carrier frequency in this Mexican population is significantly higher compared with that in a Hispanic population from the USA previously examined by Hendrickson et al (4). In the northeastern Mexican population, the frequency detected was $1 / 32(3.14 \%)$.

In Hispanic populations in the USA, the SMA carrier frequency is comparatively low, at 1/125 (24,25). However, the term Hispanic is used as a general term to refer to all persons of Hispanic descent and encompasses a diverse group of individuals (26). A previous study of 7,655 Hispanic individuals in the USA, in which family history and ethnicity was considered (27), observed that SMN1 one-copy frequency did not differ significantly from that observed in an existing study of 1,030 individuals $(\mathrm{P}=0.1869)$ (4). A recent study demonstrated that the Mexican population is genetically diverse. Therefore, detailed studies of population structure, including geographical data, may be required in order to assess the frequency and prevalence of genetic diseases in native and Mestizo Mexican populations (28).

The universal implementation of expanded newborn screenings for metabolic inherited diseases has been tailored to specific populations and countries. If the carrier status of an individual for a genetic disease is known, several options are available: i) Choice of a partner who is a non-carrier; ii) pre-implantation diagnosis; iii) prenatal diagnosis; or iv) acceptance of the $25 \%$ probability of a child being affected. In cases of infants born affected by the disease, early recognition allows for more effective treatment, which justifies the use of SMA as a target disease for population screening $(6,29,30)$. The identification of SMA carriers may facilitate a reduction in the prevalence of SMA.

To the best of our knowledge, the frequency of SMA carriers in the Mexican populations investigated in the present study has not been reported previously. A carrier frequency of $1 / 38(2.62 \%)$ was determined in the present study. No significant differences in carrier frequency were identified between populations from the northeastern and central regions of Mexico. Therefore, the frequency of the deletion of the SMN1 gene remains homogeneous across the Mexican populations examined. Further studies with larger sample sizes may provide an improved understanding of whether this frequency distribution is representative of the entire Mexican population; however, this pilot study provides an initial approximation of the carrier frequency for Mexican individuals. Furthermore, these results may be used to improve evaluation procedures, and thus justify future studies on a larger scale to validate neonatal screening in the Mexican population. It is hypothesized that carrier testing may be a useful technique to prevent SMA, which frequently results in mortality and is currently untreatable. Similar studies have been conducted in other populations, including Israeli, Caucasian, Ashkenazi Jewish, Asian and African-American populations (Table II).
The present study is relevant to the study of carrier and patient frequencies in mixed populations of complex ethnic components. The Mexican population is a convenient model for genetic studies due to its notable ethnic diversity in native and Mestizo populations. A previous study reported significantly different ancestry between individuals from separate geographic regions in Mexico (28). In order to avoid errors such as stratification, false negatives and irreproducibility, the results of the present study may be used as a reference in genetic studies of gene frequency, prevalence and association in characterized populations. In the present study of SMA carrier frequency in the northeastern and central Mexico populations, no statistically significant differences were observed; however, further studies are required to evaluate the remaining regions of Mexico.

The technique applied in the present study is an alternative to existing molecular tests to determine the copy number of the SMN1 gene in order to detect SMA carriers and affected individuals.

\section{Acknowledgements}

The authors acknowledge the medical students Alan Ureña, Dolores Álvarez and Fabiola Yazmín Agüero Zapata for their collaborative contribution in recovering patient samples, Mauricio de la Rosa Garza for technical support, and Celia Nohemi Sanchez Dominguez for critically reviewing the manuscript. The authors thank Dr Guillermo Barrera Jr for database management. The present study was partially funded by a grant from CONACYT/2008/SNI and by the Research Department of the Autonomous University of Nuevo León. American Journal Experts reviewed the grammar of the manuscript.

\section{References}

1. Markowitz JA, Singh P and Darras BT: Spinal muscular atrophy: A clinical and research update. Pediatr Neurol 46: 1-12, 2012.

2. Scheffer H, Cobben JM, Matthijs G and Wirth B: Best practice guidelines for molecular analysis in spinal muscular atrophy. Eur J Hum Genet 9: 484-491, 2001.

3. Prior TW: Perspectives and diagnostic considerations in spinal muscular atrophy. Genet Med 12: 145-152, 2010.

4. Hendrickson BC, Donohoe C, Akmaev VR, et al: Differences in SMN1 allele frequencies among ethnic groups within North America. J Med Genet 46: 641-644, 2009.

5. Jedrzejowska M, Milewski M, Zimowski J, et al: Phenotype modifiers of spinal muscular atrophy: The number of SMN2 gene copies, deletion in the NAIP gene and probably gender influence the course of the disease. Acta Biochim Pol 56: 103-108, 2009.

6. Monani UR and De Vivo DC: Neurodegeneration in spinal muscular atrophy: From disease phenotype and animal models to therapeutic strategies and beyond. Future Neurol 9: 49-65, 2014.

7. Ogino S, Leonard DG, Rennert H, Ewens WJ and Wilson RB: Genetic risk assessment in carrier testing for spinal muscular atrophy. Am J Med Genet 110: 301-307, 2002.

8. Majumdar R, Rehana Z, Al Jumah M and Fetaini N: Spinal muscular atrophy carrier screening by multiplex polymerase chain reaction using dried blood spot on filter paper. Ann Hum Genet 69: 216-221, 2005.

9. Hasanzad M, Golkar Z, Kariminejad R, et al: Deletions in the survival motor neuron gene in Iranian patients with spinal muscular atrophy. Ann Acad Med Singapore 38: 139-141, 2009.

10. Hasanzad M, Azad M, Kahrizi K, et al: Carrier frequency of SMA by quantitative analysis of the SMN1 deletion in the Iranian population. Eur J Neurol 17: 160-162, 2010.

11. Dobrowolski SF, Pham HT, Downes FP, Prior TW, Naylor EW and Swoboda KJ: Newborn screening for spinal muscular atrophy by calibrated short-amplicon melt profiling. Clin Chem 58: 1033-1039, 2012 
12. Wirth B, Herz M, Wetter A, et al: Quantitative analysis of survival motor neuron copies: Identification of subtle SMN1 mutations in patients with spinal muscular atrophy, genotype-phenotype correlation and implications for genetic counseling. Am J Hum Genet 64: 1340-1356, 1999.

13. Tizzano E and Baiget M: Molecular basis of spinal muscular atrophy: The SMN gene. Neurologia 15: 393-400, 2000 (In Spanish).

14. Sumner CJ: Therapeutics development for spinal muscular atrophy. NeuroRx 3: 235-245, 2006.

15. Singh NN and Singh RN: Alternative splicing in spinal muscular atrophy underscores the role of an intron definition model. RNA Biol 8: 600-606, 2011.

16. Yu-Jin Q, Juan D, Er-zhen L, et al: Subtle mutations in the SMNI gene in Chinese patients with SMA: p.Arg288Met mutation causing SMN1 transcript exclusion of exon7. BMC Med Genet 13: $86,2012$.

17. Monani UR, McPherson JD and Burghes AH: Promoter analysis of the human centromeric and telomeric survival motor neuron genes (SMNC and SMNT). Biochim Biophys Acta 1445: 330-336, 1999.

18. Anhuf D, Eggermann T, Rudnik-Schöneborn S and Zerres K: Determination of SMN1 and SMN2 copy number using TaqMan technology. Hum Mutat 22: 74-78, 2003.

19. Yoon S, Lee $\mathrm{CH}$ and Lee KA: Determination of SMN1 and SMN2 copy numbers in a Korean population using multiplex ligation-dependent probe amplification. Korean J Lab Med 30: 93-96, 2010.

20. Ye S, Dhillon S, Ke X, Collins AR and Day IN: An efficient procedure for genotyping single nucleotide polymorphisms. Nucleic Acids Res 29: E88-E88, 2001.

21. Cheng CY, Kao WH, Patterson N, et al: Admixture mapping of 15,280 African Americans identifies obesity susceptibility loci on chromosomes 5 and X. PLoS Genet 5: e1000490, 2009.
22. Guthrie PA, Gaunt TR, Abdollahi MR, et al: Amplification ratio control system for copy number variation genotyping. Nucleic Acids Res 39: e54, 2011

23. Ben-Shachar S, Orr-Urtreger A, Bardugo E, Shomrat R and Yaron Y: Large-scale population screening for spinal muscular atrophy: Clinical implications. Genet Med 13: 110-114, 2011.

24. Wirth B: An update of the mutation spectrum of the survival motor neuron gene $(S M N 1)$ in autosomal recessive spinal muscular atrophy (SMA). Hum Mutat 15: 228-237, 2000.

25. Muralidharan K, Wilson RB, Ogino S, Nagan N, Curtis C and Schrijver I: Population carrier screening for spinal muscular atrophy a position statement of the association for molecular pathology. J Mol Diagn 13: 3-6, 2011.

26. Siegel R, Naishadham D and Jemal A: Cancer statistics for Hispanics/Latinos, 2012. CA: Cancer J Clin 62: 283-298, 2012.

27. Sugarman EA, Nagan N, Zhu H, et al: Pan-ethnic carrier screening and prenatal diagnosis for spinal muscular atrophy: Clinical laboratory analysis of $>72,400$ specimens. Eur J Hum Genet 20: 27-32, 2012

28. Moreno-Estrada A, Gignoux CR, Fernández-López JC, et al Human genetics. The genetics of Mexico recapitulates Native American substructure and affects biomedical traits. Science 344: 1280-1285, 2014

29. Basel-Vanagaite L, Taub E, Drasinover V, et al: Genetic carrier screening for spinal muscular atrophy and spinal muscular atrophy with respiratory distress 1 in an isolated population in Israel. Genet Test 12: 53-56, 2008.

30. Arkblad E, Tulinius M, Kroksmark AK, Henricsson M and Darin N: A population-based study of genotypic and phenotypic variability in children with spinal muscular atrophy. Acta Paediatr 98: 865-872, 2009. 